\title{
Hábito de pesquisar sobre saúde na internet entre idosos atendidos na Atenção Primária à Saúde: prevalência e fatores associados
}

\author{
Habit of investigating health information on the internet among the elderly population \\ attended in Primary Health Care: prevalence and associated factors \\ Hábito de investigar la salud en internet en personas mayores atendidas en Atención \\ Primaria de Salud: prevalencia y factores asociados
}

Tasso Kfuri Araújo Mafra ${ }^{1 *}$, Jéssica Pasquali Kasperavicius ${ }^{1}$, Luan Silva Franceschetto², Lucimar Maria Fossatti de Carvalho' ${ }^{1}$, Lissandra Glusczak ${ }^{1}$, Regina Inês Kunz ${ }^{1}$, Jossimara Polettini ${ }^{1}$, Shana Ginar da Silva1', Gustavo Olszanski Acrani'1, Ivana Loraine Lindemann¹.

\section{RESUMO}

Objetivo: Avaliar prevalência e fatores associados ao hábito de pesquisar sobre saúde na internet entre idosos usuários da Atenção Primária à Saúde (APS). Métodos: Estudo transversal realizado de maio a agosto de 2019, no norte do Rio Grande do Sul, com aplicação de questionário sobre características sociodemográficas, de saúde e comportamento. A estatística incluiu descrição da amostra e cálculo da prevalência do desfecho com intervalo de confiança de 95\% (IC95). A verificação das associações entre desfecho e variáveis preditoras foi realizada por Regressão de Poisson. Na análise bivariada, calcularam-se as Razões de Prevalência (RP) brutas e seus IC95 e na multivariada, com variância robusta para conglomerados, as RP ajustadas e seus IC95. Resultados: Na amostra $(n=403)$, a prevalência do desfecho foi de 19\% (IC95 15-22), com diferença significativa em relação às variáveis sexo ( $R P=2,14 ; 1,29-3,55)$, cor da pele $(R P=1,82 ; 1,04-3,19)$, renda $(R P=1,61 ; 1,08-2,42)$, escolaridade $(R P=4,04 ; 2,48-6,59)$, tempo desde a última consulta médica na APS ( $R P=2,55 ; 1,61-4,04)$ e consumo de bebida alcoólica $(R P=0,46 ; 0,32-0,68)$. Conclusão: Aproximadamente um quinto dos idosos utilizam a internet para pesquisar sobre saúde. Assim, é importante que os profissionais de saúde atentem a tal comportamento entre as pessoas atendidas, visando orientá-las para o uso adequado da ferramenta.

Palavras-chave: Atenção Primária à Saúde, Idoso, Internet.

\section{ABSTRACT}

Objective: To assess the prevalence and the associated factors to the habit of researching health information on the internet among elderly users of Primary Health Care (PHC). Methods: Cross-sectional study carried out in the north of Rio Grande do Sul with application of questionnaire containing sociodemographic, health and behavioral characteristics. Statistics included description of the sample and calculation of the prevalence of the outcome with $95 \%$ confidence interval (C195). The verification of associations between outcome and predictor variables were performed by Poisson Regression. In the bivariate analysis, the crude Prevalence Ratios (PR) and their IC95 were calculated, while in the multivariate, with robust variance for clusters, the adjusted PRs and their IC95 were calculated. Results: In the sample $(n=403)$, the prevalence of the outcome was $19 \%$ (CI95 15-22), with a significant difference in relation to gender ( $P R=2.14 ; 1.29-3.55)$, color skin $(P R=1.82 ; 1.04-3.19)$, income ( $P R=1.61 ; 1.08-2.42)$, education ( $P R=4.04 ; 2.48-6.59)$, time since the last medical appointment at the PHC (PR=2.55; 1.61-4.04) and alcohol consumption ( $P R=0.46 ; 0.32-0.68)$.

1 Universidade Federal da Fronteira Sul (UFFS), Passo Fundo - RS. *E-mail: tassokfuri@gmail.com

2 Instituto Meridional (IMED), Passo Fundo - RS. 
Conclusion: Approximately one fifth use the internet to research about health. Thus, it is important that health professionals pay attention to this behavior, aiming to guide the PHC users in the proper use of the tool.

Keywords: Primary Health Care, Elderly, Internet.

\section{RESUMEN}

Objetivo: Evaluar la prevalencia y los factores asociados al hábito de investigar la salud en internet entre los usuarios ancianos de la Atención Primaria de Salud (APS). Métodos: Estudio transversal con la aplicación de un cuestionario conteniendo preguntas sociodemográficas, de salud y comportamiento. Se realizaron estadísticas descriptivas y la verificación de las asociaciones entre el resultado y las variables predictoras mediante Regresión de Poisson. En el análisis bivariado se calcularon las Razones de Prevalencia (RP) brutas y su IC95, y en el multivariado, con varianza robusta por conglomerados, las RP ajustadas y su IC95. Resultados: En la muestra $(n=403)$, la prevalencia del variable dependiente fue 19\% (IC95 15-22), con diferencia significativa en relación a sexo $(R P=2,14 ; 1,29-3,55)$, color de piel $(R P=1,82 ; 1,04-3,19)$, ingreso $(\mathrm{RP}=1,61 ; 1,08-2,42)$, educación $(\mathrm{RP}=4,04 ; 2,48-6,59)$, tiempo desde la última consulta médica en APS $(\mathrm{RP}=2,55 ; 1,61-4,04)$ y consumo de alcohol $(\mathrm{PR}=0,46 ; 0,32-0,68)$. Conclusión: Aproximadamente una quinta parte utiliza internet para investigar sobre la salud. Por lo tanto, es importante que los profesionales de la salud presten atención a este comportamiento entre las personas atendidas para orientarlos en el uso adecuado de la herramienta.

Palabras clave: Atención Primaria de Salud, Anciano, Internet.

\section{INTRODUÇÃO}

No Brasil, o número de pessoas com idade igual ou superior a 60 anos, no início do século XXI, era estimado em 20 milhões de pessoas. Em 2017, o país superou a marca de 30, 2 milhões de idosos, conforme a pesquisa Nacional por Amostra de Domicílios (PNAD) de 2018, sendo esse aumento reflexo da maior expectativa de vida, redução das taxas de fecundidade e das melhores condições de saúde da população. Nessa perspectiva, estima-se que o número de pessoas idosas esteja próximo de 66,5 milhões em 2050 (INSTITUTO BRASILEIRO DE GEOGRAFIA E ESTATíSTICA (IBGE), 2018a). Esse novo contexto demográfico, dada a carga de doenças crônicas e incapacidades esperadas em populações mais envelhecidas, representa um desafio ao sistema de saúde à medida que aumenta a procura pelos serviços e exige maior capacidade de responder às demandas em termos de coordenação do cuidado e qualidade da assistência (PAIM J, et al., 2011; WANG L, et al., 2021).

Frente a esse cenário e considerando a complexidade atual das necessidades em saúde, as Tecnologias de Informação e Comunicação (TIC), ainda que incipientes, configuram-se como uma importante ferramenta no apoio aos processos de cuidado. Dentre as potencialidades destacam-se a qualidade da interface com os usuários, funcionalidade, eficiência de comunicação, gestão de cuidados no contexto da prática clínica e resultados na atenção aos pacientes crônicos (SANTOS AF, et al., 2017). Diante da crescente expansão e usabilidade da internet, essa ferramenta, por sua abrangência e interatividade, tem sido útil para propagação de informações direcionadas aos idosos, principalmente de saúde, compondo um instrumento eficiente para auxílio de políticas de saúde pública (GUSTAFSON SR DH, et al., 2015; MIRANDA LM e FARIAS SF, 2009).

Por outro lado, faz-se necessário destacar as marcantes desigualdades no acesso e no uso da internet no Brasil dado aos múltiplos contextos econômicos e sociais, sua complexidade e multideterminação (CÔMITE GESTOR DA INTERNET NO BRASIL (CGI.BR), 2019; NETO AP e FLYNN MB, 2021). Ainda assim, estudos demonstram que os idosos, cada vez mais, buscam por informações atuais e pela informatização, estimulados pela vontade de sentirem-se inseridos em um grupo social (MIRANDA LM e FARIAS SF, 2009; MIRANDA GMD, et al., 2016; FERREIRA MC e TEIXEIRA KMD, 2017). Nesse contexto, o uso da internet traz benefícios ao convívio social, enfrentamento das situações de vida, conhecimento sobre doenças, suporte emocional, qualidade de vida e manutenção da capacidade cognitiva (NEVES BB, et al., 2018; CASADEI GR, et al., 2019; GUSTAFSON SR DH, et al., 2021a; KRUG RR, et al., 2019). 
$\mathrm{Na}$ área da saúde, a interação pela internet possibilita a troca de experiências entre indivíduos com dificuldades semelhantes e facilita o debate, sendo indicada, por alguns, como uma boa estratégia para manejar diversas condições clínicas (MURRAY E, et al., 2005). O acesso às redes possibilitou o surgimento do "paciente expert", usuário da saúde que busca informações na internet, o que tem alterado a relação médico-paciente, na qual este assume um papel mais participativo no cuidado à saúde (KNORST GRS, et al., 2019).

Entretanto, o acesso à quantidade de informações não direcionadas e excessivas pode levar esse paciente a uma menor disponibilidade em acatar as recomendações dos profissionais de saúde. Comumente o usuário pode ter dificuldade de distinguir os conteúdos, os termos médicos e a veracidade de informações (GARBIN HBR, et al., 2008). Nesse contexto, programas virtuais de assistência à saúde do idoso, com atividades direcionadas e supervisionadas, a exemplo do ElderTree norte-americano, têm demonstrado resultados positivos em aliviar a sobrecarga no sistema de atenção primária, especialmente entre usuários com doenças crônicas (GUSTAFSON SR DH, 2021b).

Apesar da internet ter um crescimento exponencial nas últimas décadas e sendo instrumento central na organização de novas políticas e processos, não foram identificados muitos estudos na área da saúde sobre os comportamentos de idosos no Brasil. Desta forma, este estudo teve como objetivo avaliar tal temática entre usuários da Atenção Primária à Saúde (APS) de uma cidade de porte médio no norte do Rio Grande do Sul, Brasil.

\section{MÉTODOS}

O presente estudo é um recorte de uma pesquisa transversal realizada em uma cidade no norte do Rio Grande do Sul, a qual aborda características sociodemográficas, de saúde e de comportamento de usuários da Atenção Primária à Saúde (APS). A pesquisa original teve o cálculo amostral calculado de duas formas, nas quais considerou-se nível de significância de $95 \%$ e poder de estudo de $80 \%$. A fim de identificar uma prevalência de desfecho de $10 \%$ realizou-se o primeiro cálculo, com margem de erro de cinco pontos percentuais, resultando em 138 participantes. Para identificar a associação entre os diferentes desfechos e fatores de exposição, realizou-se o segundo cálculo, tendo como base uma razão de não expostos/expostos de 9:1, prevalência total do desfecho de 10\%, frequência esperada em não expostos de $9,1 \%$ e Razão de Prevalências (RP) de 2 . O cômputo resultou em 1.220 entrevistados e, somando-se a esse número 15\% para fatores de confusão, a amostra final mínima seria de 1.403 participantes.

Na seleção da amostra, foram incluídas todas as 34 unidades urbanas da APS e número de entrevistados em cada uma foi estabelecido mantendo-se a proporção em relação à quantidade de procedimentos realizados no mês que antecedeu o início da coleta de dados. Posteriormente, todos os usuários que estavam na unidade, a espera de realizar algum procedimento, foram incluídos de forma consecutiva, até que fosse alcançado o número estipulado ou até que todos os usuários presentes no último turno da coleta em cada unidade fossem convidados a participar. Um questionário testado e codificado foi o instrumento utilizado por entrevistadores treinados, o qual foi aplicado entre maio e agosto de 2019. Idade igual ou superior a 18 anos, ambos os sexos, residência no município e condições de responder ao questionário foram considerados critérios de elegibilidade. Para este estudo foram excluídos os adultos.

O desfecho, hábito de pesquisar sobre saúde na internet, foi aferido através da pergunta: Você tem o costume de acessar a internet? Para aqueles que responderam positivamente, foi questionado: Você tem o costume de pesquisar sobre saúde na internet? O desfecho foi considerado positivo para as respostas sempre/às vezes e negativo para não/nunca. Aos que responderam afirmativamente, foi ainda perguntado se "Você acredita no que encontra sobre saúde na internet? e "Você comenta com o médico sobre o que encontra sobre saúde na internet?", tendo como opções de resposta para ambas: sempre; às vezes e; não/nunca.

Para a análise das exposições, foram consideradas as variáveis sociodemográficas sexo (masculino; feminino), idade em anos completos $(60-69 ; \geq 70)$, cor da pele autorreferida (branca; outra), renda familiar per capita ( $\leq 1$ salário mínimo; >1 salário mínimo), escolaridade (ensino fundamental; médio; superior) e morar sozinho (não; sim). 
Em relação à saúde foram avaliadas multimorbidade (diagnóstico médico autorreferido de duas ou mais doenças crônicas não transmissíveis: obesidade, diabetes mellitus, hipertensão arterial sistêmica, hipercolesterolemia, hipertrigliceridemia e cardiopatia), dor crônica (dor há no mínimo seis meses), polifarmácia (uso diário e concomitante de cinco ou mais fármacos) (MASNOON N, et al., 2017). Também foi analisado a ideação suicida (sim; não) e a insônia, avaliada a partir da presença de dois dos quatro critérios: apresentar dificuldades em pegar no sono, acordar de madrugada e ter dificuldades para voltar a dormir, dormir menos de 6 horas por noite e se sentir cansado durante o dia por não ter dormido bem (LEGER D, et al., 2000).

O estado nutricional, conforme Lipschitz DA (1994), foi analisado de acordo com o Índice de Massa Corporal (IMC) (com sobrepeso; sem sobrepeso), o tempo em meses desde a última consulta médica na APS $(\leq 1 ; 2-6 ; 7-12 ;>12)$, a autopercepção da saúde e da alimentação (positiva; negativa) e a autopercepção de risco a infecções sexualmente transmissíveis (sim; não).

Por fim, as variáveis comportamentais analisadas foram automedicação nos últimos 30 dias, vacinação contra influenza no último ano, consumo de bebidas alcoólicas, tabagismo e prática de atividade física no lazer (sim; não). Além disso, foi avaliada a alimentação com base nos marcadores do consumo alimentar estabelecidos pelo Ministério da Saúde (MINISTÉRIO DA SAÚDE, 2016). Foram considerados como adequados os hábitos alimentares de indivíduos que responderam afirmativamente para o consumo de feijão, de frutas frescas e de verduras e/ou legumes no dia anterior e, negativamente para o consumo de hambúrger e/ou embutidos; de bebidas adoçadas; de macarrão instantâneo, salgadinhos de pacote ou biscoitos salgados e; de biscoito recheado, doces ou guloseimas.

Após dupla digitação e validação, a estatística incluiu a descrição da amostra e o cálculo da prevalência do desfecho com intervalo de confiança de 95\% (IC95\%). A verificação das associações entre desfecho e variáveis preditoras foi realizada por Regressão de Poisson. $\mathrm{Na}$ análise bivariada, calcularam-se as Razões de Prevalência (RP) brutas e seus IC95 e na multivariada, com variância robusta para conglomerados, as RP ajustadas e seus IC95. A análise multivariada foi do tipo backward stepwise, seguindo um modelo hierárquico pré-estabelecido (VICTORA CG, et al., 1997). Foram adotados três níveis de determinação (1ํnível variáveis sociodemográficas; $2^{\circ}$ nível condições de saúde; $3^{\circ}$ nível variáveis comportamentais), sendo que em cada nível as variáveis foram ajustadas entre si e aquelas que apresentaram $p \leq 0,20$ foram mantidas para ajuste com o nível seguinte.

Nas variáveis categóricas politômicas, devido ao ordenamento entre as categorias, foi realizado o teste de Wald para tendência linear. Em todos os testes, foi admitido erro $\alpha$ de $5 \%$, sendo considerados significativos valores de $p<0,05$, para testes bicaudais, por meio do software Stata/IC 11.2 (StataCorp, College Station, Texas, USA, serial number 30110589642).

O protocolo do estudo foi aprovado pelo Comitê de Ética em Pesquisa com Seres Humanos da Universidade Federal da Fronteira Sul (CAAE 09474719.3.0000.5564), obedecendo à Resolução no 466/2012 do Conselho Nacional de Saúde e a todos os participantes foi solicitado o Termo de Consentimento Livre e Esclarecido (TCLE).

\section{RESULTADOS}

A amostra foi composta por 403 idosos e sua caracterização sociodemográfica, de saúde e de comportamento está apresentada na Tabela 1 . Observou-se que 61,3\% eram mulheres, $66,5 \%$ tinham entre 60 e 69 anos, $67,6 \%$ se declararam brancos, $61,4 \%$ apresentavam renda per capita de até um salário mínimo, $64,7 \%$ referiram ensino fundamental e $79,9 \%$ não moravam sozinhos.

Quanto às características de saúde, $57,1 \%$ apresentaram excesso de peso, 68,2\% multimorbidade, 43,9\% dor crônica, 32,8\% polifarmácia, $80,7 \%$ insônia e 19,8\% histórico de ideação suicida. Ainda, 45,7\% dos participantes mencionaram ter realizado consulta médica na APS no mês anterior à entrevista. A autopercepção da saúde foi relatada como negativa em $63,1 \%$ dos idosos, bem como a autopercepção negativa da alimentação em $24,6 \%$, enquanto $84,5 \%$ não consideravam apresentar comportamento de risco para infecções sexualmente transmissíveis (Tabela 1). 
Tabela 1 - Caracterização sociodemográfica, de saúde e de comportamento de idosos atendidos na Atenção Primária à Saúde (APS), 2019 ( $\mathrm{n=403).}$

\begin{tabular}{|c|c|c|}
\hline Variáveis & $\mathbf{n}$ & $\%$ \\
\hline \multicolumn{3}{|l|}{ Sexo } \\
\hline $\begin{array}{l}\text { Masculino } \\
\text { Feminino }\end{array}$ & $\begin{array}{l}156 \\
247\end{array}$ & $\begin{array}{l}38,7 \\
61,3\end{array}$ \\
\hline \multicolumn{3}{|c|}{ Faixa etária em anos completos } \\
\hline $\begin{array}{l}60-69 \\
\geq 70\end{array}$ & $\begin{array}{l}268 \\
135 \\
\end{array}$ & $\begin{array}{l}66,5 \\
33,5 \\
\end{array}$ \\
\hline \multicolumn{3}{|l|}{ Cor da pele $(n=398)$} \\
\hline $\begin{array}{l}\text { Branca } \\
\text { Outra }\end{array}$ & $\begin{array}{l}269 \\
129 \\
\end{array}$ & $\begin{array}{l}67,6 \\
32,4 \\
\end{array}$ \\
\hline \multicolumn{3}{|c|}{ Renda familiar mensal per capita* $(n=373)$} \\
\hline $\begin{array}{l}\leq 1 \text { salário mínimo } \\
>1 \text { salário mínimo }\end{array}$ & $\begin{array}{l}229 \\
144 \\
\end{array}$ & $\begin{array}{l}61,4 \\
38,6 \\
\end{array}$ \\
\hline \multicolumn{3}{|l|}{ Escolaridade $(n=343)$} \\
\hline $\begin{array}{l}\text { Ensino fundamental } \\
\text { Ensino médio } \\
\text { Ensino superior ou mais }\end{array}$ & $\begin{array}{l}222 \\
73 \\
48\end{array}$ & $\begin{array}{l}64,7 \\
21,3 \\
14,0\end{array}$ \\
\hline \multicolumn{3}{|l|}{ Morar sozinho } \\
\hline $\begin{array}{l}\text { Sim } \\
\text { Não }\end{array}$ & $\begin{array}{c}81 \\
322\end{array}$ & $\begin{array}{l}20,1 \\
79,9\end{array}$ \\
\hline \multicolumn{3}{|l|}{ Estado nutricional $(\mathrm{n}=347)$} \\
\hline $\begin{array}{l}\text { Com sobrepeso } \\
\text { Sem sobrepeso }\end{array}$ & $\begin{array}{l}198 \\
149\end{array}$ & $\begin{array}{l}57,1 \\
42,9\end{array}$ \\
\hline \multicolumn{3}{|l|}{ Multimorbidade } \\
\hline $\begin{array}{l}\text { Sim } \\
\text { Não } \\
\end{array}$ & $\begin{array}{l}275 \\
128 \\
\end{array}$ & $\begin{array}{l}68,2 \\
31,8 \\
\end{array}$ \\
\hline \multicolumn{3}{|l|}{ Dor crônica } \\
\hline $\begin{array}{l}\text { Sim } \\
\text { Não }\end{array}$ & $\begin{array}{l}177 \\
226 \\
\end{array}$ & $\begin{array}{l}43,9 \\
56,1\end{array}$ \\
\hline \multicolumn{3}{|l|}{ Polifarmácia } \\
\hline $\begin{array}{l}\text { Sim } \\
\text { Não }\end{array}$ & $\begin{array}{l}132 \\
271\end{array}$ & $\begin{array}{l}32,8 \\
67,2\end{array}$ \\
\hline \multicolumn{3}{|l|}{ Insônia (n=399) } \\
\hline $\begin{array}{l}\text { Sim } \\
\text { Não }\end{array}$ & $\begin{array}{c}322 \\
77 \\
\end{array}$ & $\begin{array}{l}80,7 \\
19,3 \\
\end{array}$ \\
\hline \multicolumn{3}{|l|}{ Tdeação suicida } \\
\hline $\begin{array}{l}\text { Simim } \\
\text { Não }\end{array}$ & $\begin{array}{c}80 \\
323\end{array}$ & $\begin{array}{l}19,8 \\
80,2\end{array}$ \\
\hline \multicolumn{3}{|c|}{ Tempo em meses desde a última consulta médica na APS $(n=398)$} \\
\hline $\begin{array}{l}\leq 1 \\
2-6 \\
7-12 \\
>12 \\
\end{array}$ & $\begin{array}{l}182 \\
151 \\
51 \\
14\end{array}$ & $\begin{array}{l}45,7 \\
38,0 \\
12,8 \\
3,5\end{array}$ \\
\hline \multicolumn{3}{|c|}{ Autopercepção da saúde ( $n=401)$} \\
\hline $\begin{array}{l}\text { Positiva } \\
\text { Negativa }\end{array}$ & $\begin{array}{l}148 \\
253\end{array}$ & $\begin{array}{l}36,9 \\
63,1\end{array}$ \\
\hline \multicolumn{3}{|c|}{ Autopercepção da alimentação } \\
\hline $\begin{array}{l}\text { Positiva } \\
\text { Negativa }\end{array}$ & $\begin{array}{c}304 \\
99\end{array}$ & $\begin{array}{l}75,4 \\
24,6\end{array}$ \\
\hline \multicolumn{3}{|c|}{ Autopercepção de risco a infecçóes sexualmente transmissiveis ( $\mathrm{n}=206$ ) } \\
\hline Sim & $\begin{array}{l}32 \\
174\end{array}$ & $\begin{array}{l}15,5 \\
84,5\end{array}$ \\
\hline \multicolumn{3}{|c|}{ Automedicação nos últimos 30 dias ( $n=401$ ) } \\
\hline $\begin{array}{l}\text { Sim } \\
\text { Não }\end{array}$ & $\begin{array}{l}176 \\
225\end{array}$ & $\begin{array}{l}43,9 \\
56,1\end{array}$ \\
\hline \multicolumn{3}{|c|}{ Vacinação contra a gripe no último ano } \\
\hline$\underset{\text { São }}{\operatorname{Sim}}$ & $\begin{array}{c}317 \\
86\end{array}$ & $\begin{array}{l}78,7 \\
21,3\end{array}$ \\
\hline \multicolumn{3}{|c|}{ Consumo de bebida alcoólica $(n=402)$} \\
\hline $\begin{array}{l}\text { Sim } \\
\text { Não }\end{array}$ & $\begin{array}{l}95 \\
307\end{array}$ & $\begin{array}{l}23,6 \\
76,4\end{array}$ \\
\hline \multicolumn{3}{|l|}{ Tabagismo $(\mathrm{n}=402)$} \\
\hline $\begin{array}{l}\text { Sim } \\
\text { Não }\end{array}$ & $\begin{array}{c}57 \\
345 \\
\end{array}$ & $\begin{array}{l}14,2 \\
85,8\end{array}$ \\
\hline \multicolumn{3}{|l|}{ Prática de atividade fisica } \\
\hline $\begin{array}{l}\text { Sim } \\
\text { Não }\end{array}$ & $\begin{array}{l}218 \\
185 \\
\end{array}$ & $\begin{array}{l}54,1 \\
45,9\end{array}$ \\
\hline \multicolumn{3}{|l|}{ Hábitos alimentares $(n=398)$} \\
\hline $\begin{array}{l}\text { Adequados } \\
\text { Inadequados }\end{array}$ & $\begin{array}{c}76 \\
322 \\
\end{array}$ & $\begin{array}{l}19,1 \\
80,9\end{array}$ \\
\hline
\end{tabular}

Legenda: * Salário mínimo: $\mathrm{R} \$ 998,00$. Fonte: Mafra TKA, et al., 2021.

Sobre as variáveis comportamentais, observou-se que 43,9\% se automedicaram nos 30 dias anteriores, $78,7 \%$ realizaram a vacina contra influenza no ano anterior, $23,6 \%$ consumiam bebidas alcoólicas de forma crônica ou esporádica, 14,2\% fumavam, 54,1\% praticavam atividades físicas e 19,1\% apresentavam hábitos alimentares adequados.

Do total de idosos, 19\% (IC95\%: 15-22) reportaram utilizar a internet para pesquisar sobre saúde. Daqueles que relataram este hábito, 15,8\% referiram acreditar sempre nos achados da pesquisa, 67,1\% acreditam às vezes e $17,1 \%$ não acreditam. Considerando os idosos que pesquisam sobre saúde na internet, em relação a comentar sobre os resultados das pesquisas com o médico, 13,2\% dos participantes falaram que comentam sempre, $22,4 \%$ às vezes e $64,5 \%$ nunca. 
Conforme demonstrado na Tabela 2, na análise multivariada, após o ajuste, dentre as variáveis do 1ํㅡível, sexo, cor da pele autorreferida, renda per capita e escolaridade permaneceram associadas ao desfecho. Houve maior probabilidade da utilização da internet para pesquisa sobre saúde entre as mulheres $(\mathrm{RP}=2,14$; IC95 1,29-3,55), entre os idosos com cor de pele branca ( $R P=1,82$; IC95 1,04-3,19) e de maior renda $(\mathrm{RP}=1,61$; IC95 1,08-2,42). Ainda, observou-se que quanto maior a escolaridade dos participantes, maior também a probabilidade de pesquisar sobre saúde na internet ( $R P=4,04$; IC95 2,48-6,59 entre aqueles com ensino superior).

Tabela 2 - Análise bruta e ajustada de fatores associados a pesquisa sobre saúde na internet por idosos atendidos na Atenção Primária à Saúde (APS), 2019 ( $n=403)$.

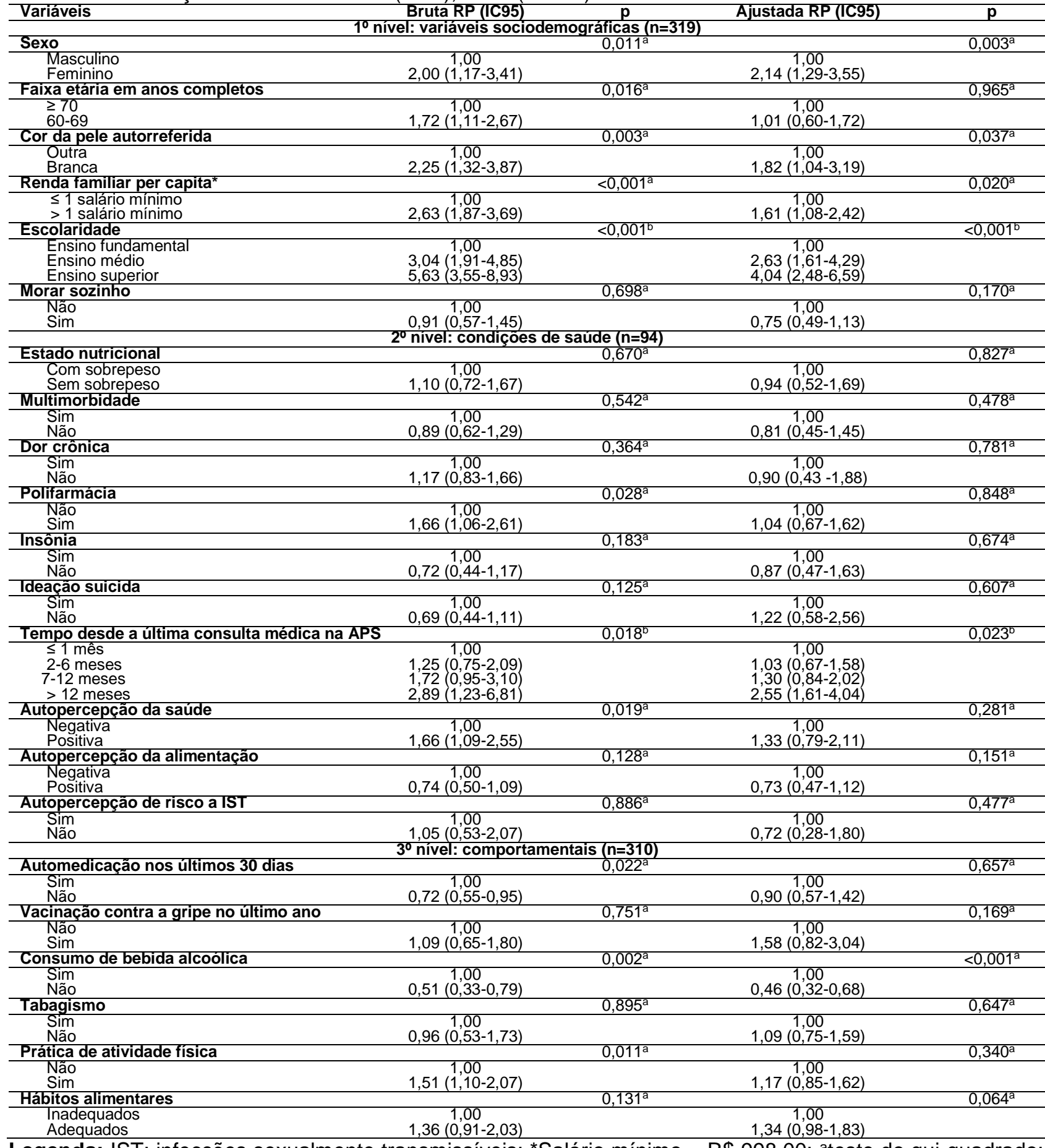

Legenda: IST: infecções sexualmente transmissíveis; *Salário mínimo = $\mathrm{R} \$ 998,00$; ateste do qui-quadrado; bteste de tendência linear. Fonte: Mafra TKA, et al., 2021. 
No 2o nível, dentre as variáveis relacionadas a condições de saúde, verificou-se associação estatisticamente significativa entre o desfecho e o tempo decorrido desde a última consulta médica na APS, sendo que, quanto maior o tempo, mais elevada a probabilidade de recorrer à internet para pesquisar sobre saúde ( $R P=2,55$; IC95 1,61-4,04) entre aqueles que consultaram há mais de 12 meses).

Em relação às variáveis mais proximais, constatou-se que entre os idosos que negaram o comportamento de consumir bebidas alcoólicas, houve redução de $54 \%$ na probabilidade de pesquisarem sobre saúde na internet (RP=0,46; IC95 0,32-0,68).

Por fim, não foram encontradas associações estatisticamente significantes entre o hábito de pesquisar sobre saúde na internet e faixa etária (60-69 anos ou $\geq 70)$, morar sozinho, estado nutricional, multimorbidade, dor crônica, polifarmácia, insônia, ideação suicida, autopercepção da saúde, da alimentação e de risco de IST, automedicação nos últimos 30 dias, vacinação contra gripe no último ano, tabagismo e prática de atividade física no lazer.

\section{DISCUSSÃO}

Este estudo determinou a prevalência do uso da internet para pesquisas sobre saúde assim como as características associadas a esse hábito em idosos usuários da APS. Observou-se que aproximadamente um quinto dos participantes utilizam a internet como um meio para pesquisar sobre saúde. Essa prevalência é inferior aos 65\% observados no estudo nacional realizado por Diniz JL, et al. (2020), aos 55,7\% encontrados por Quittschalle J, et al. (2020) na Alemanha e aos 49\% nos Estados Unidos da América (MEISCHKE H, et al., 2005).

Tal discrepância pode ser oriunda da distinção entre as amostras, assim como da abordagem para coleta de dados. Sabidamente, em países com renda populacional elevada há maior probabilidade de acesso à internet, enquanto o Brasil se configura por marcadas desigualdades no acesso e uso dessa ferramenta (FUNDAÇÃO GETÚLIO VARGAS (FGV), 2012). Considerando ainda que o presente estudo abrangeu idosos em atendimento na rede de APS e que a maioria apresentava multimorbidade $(68,2 \%)$, supõe-se que estejam em acompanhamento contínuo e, portanto, recebam informações da equipe de saúde.

Em relação aos fatores associados, neste estudo foi observada diferença estatisticamente significativa entre o hábito de pesquisar sobre saúde na internet e as variáveis sexo, cor da pele, renda, escolaridade, tempo desde a última consulta médica na APS e consumo de bebida alcoólica.

Verificou-se prevalência duas vezes maior entre as mulheres (RP 2,14; IC95 1,29-3,55), o que está em concordância com outros estudos (PANDEY SK, et al., 2003; DINIZ JL, et al., 2020; CHOI NG e DINITO DM, 2013). Este achado pode ser explicado pelo fato de os homens buscarem menos os serviços de saúde, como resultado de uma variação das necessidades entre sexos e gêneros, sendo a saúde do homem menos explorada (BIBIANO AMB, et al., 2019). Ainda, há evidências de que as mulheres dependem cada vez mais da Internet para complementar as informações de saúde recebidas de fontes tradicionais e discutir as implicações de novas descobertas com os profissionais da saúde (PANDEY SK, et al., 2003).

Observou-se também, de modo semelhante à literatura, diferenças no que concerne à escolaridade (CAGNEY KA e LAUDERDALE DS, 2002; CHOI NG e DINITTO DM, 2013; SAMKANGE-ZEEB F, et al., 2020). Da mesma maneira, Choi NG e Dinitto DM (2013), observaram diferenças relacionadas à cor da pele e, outros estudos, em relação à renda (PANDEY SK, et al., 2003; MEISCHKE H, et al., 2005). A pesquisa mostrou que quanto maior a escolaridade, maior a prevalência do desfecho (RP 4,04; IC95 2,48-6,59 entre aqueles com ensino superior) e que entre os idosos de cor de pele branca e com maior renda, a busca por informações sobre saúde na internet foi cerca de duas vezes mais frequente (RP 1,82; IC95 1,04-3,19 e RP 1,61; IC95 1,08-2,42, respectivamente), o que pode estar relacionado à facilidade no acesso e no manuseio das tecnologias. Dados sobre a população brasileira apontam que $48 \%$ dos indígenas, $55 \%$ das pessoas pretas e $57 \%$ dos pardos utilizaram o computador apenas uma vez na vida (IBGE, 2018b).

Verificou-se que o hábito de pesquisar sobre saúde na internet foi significativamente inferior entre os idosos que não consomem bebidas alcoólicas (RP 0,46; IC95 0,32-0,68). Embora a associação ainda não tenha sido 
descrita na literatura, é provável que os indivíduos que consomem bebida alcóolica busquem informações a respeito do hábito e de possíveis interações com medicamentos em uso, questionamento frequente nos atendimentos médicos. Além disso, a maioria da amostra apresentava mais de duas doenças crônicas $(68,2 \%)$ e não consumia bebida alcóolica $(76,4 \%)$, possivelmente como um autocuidado decorrente do acompanhamento contínuo no serviço de saúde, o qual pode, da mesma forma, representar maior acesso às informações sobre saúde em geral, reduzindo a procura na internet. No mesmo sentido, verificou-se que quanto maior o tempo desde a última consulta médica na APS, maior a prevalência do desfecho (RP 2,55 IC95 1,61-4,04 entre aqueles que consultaram há mais de um ano).

O uso da internet como fonte de informação, se bem direcionado, pode ajudar os idosos a melhorar sua saúde física e mental. Nesse sentido, órgãos governamentais e não governamentais têm disponibilizado informações sobre diferentes aspectos que envolvem desde a prevenção até o tratamento de doenças. Por outro lado, em meio a informações verdadeiras, circulam aquelas de conteúdo e origem duvidosas, as quais podem prejudicar a saúde da população, interrompendo tratamentos, promovendo medidas curativas inadequadas e causando efeitos adversos (CUNHA WT, 2020).

Por fim, como ponto positivo do estudo, destaca-se a elucidação da prevalência e dos fatores associados ao hábito de pesquisar sobre saúde na internet entre a população idosa atendida na APS, tema pouco investigado no cenário nacional. Assim, é importante que as equipes de saúde atentem a tal comportamento, visando orientar os usuários para o uso adequado da ferramenta. Como limitação, cabe mencionar a natureza transversal do estudo, na qual existe possibilidade de viés de causalidade reversa entre algumas variáveis. Ainda, considerando que o estudo foi realizado como um recorte de uma pesquisa mais ampla, sendo a amostra constituída somente pelos idosos, cabe admitir a possibilidade de não terem sido encontradas associações entre algumas das variáveis independentes e o desfecho por poder estatístico insuficiente.

\section{CONCLUSÃO}

Embora neste estudo a prevalência do uso da internet para pesquisar sobre saúde tenha sido inferior à literatura, este é um hábito que possivelmente se tornará cada vez mais frequente, devido ao aumento da população idosa brasileira, e está associado ao sexo feminino, cor da pele branca, maior renda e escolaridade, maior tempo desde a última consulta médica e mais frequente em idosos que consomem bebida alcoólica. Destaca-se que a APS pode desempenhar um papel importante como agente de inclusão e de educação digital, orientando sobre o acesso a fontes confiáveis e fomentando o uso da internet como ferramenta auxiliar no cuidado à saúde. Ainda, salienta-se a necessidade de estudos adicionais que visem investigar as fontes de busca utilizadas bem como os assuntos mais pesquisados.

\section{AGRADECIMENTOS}

Os autores agradecem à Secretaria Municipal de Saúde do município, pela autorização para a realização do estudo e a todos os acadêmicos do Curso de Medicina da Universidade Federal da Fronteira Sul, que participaram da coleta e do processamento dos dados.

\section{REFERÊNCIAS}

1. BIBIANO A, et al. Fatores associados à utilização dos serviços de saúde por homens idosos: uma revisão sistemática da literatura. Ciênc. saúde colet., 2019; 24(6).

2. CAGNEY KA, LAUDERDALE DS. Education, Wealth, and Cognitive Function in Later Life. The Journals of Gerontology Series B, 2002; 57(2): 163-172.

3. CASADEI G, et al. Influência das redes sociais virtuais na saúde dos idosos. Enciclopédia Biosfera, 2019; 16(29): 1962-1975.

4. CHOI NG, DINITTO DM. Internet use among older adults: Association with health needs, psychological capital, and social capital. Journal of Medical Internet Research, 2013; 15(5): e97.

5. CÔMITE GESTOR DA INTERNET NO BRASIL (CGI.BR). Survey on the use of information and communication technologies in brazilian households. 1. ed. São Paulo: Comitê Gestor da Internet no Brasil, 2019. Disponível em: https://cetic.br/media/docs/publicacoes/2/20201123121817/tic_dom_2019_livro_eletronico.pdf. Acesso em: 7 set. 2021. 
6. CUNHA WT. Fake News: as consequências negativas para a saúde da população. Revista Baiana de Saúde Pública, 2020; 44(1): 81-102, 2020.

7. DINIZ JL, et al. Digital inclusion and Internet use among older adults in Brazil: a cross-sectional study. Revista brasileira de enfermagem, 2020; 73: e20200241.

8. FERREIRA MC, TEIXEIRA KMD. O uso de redes sociais virtuais pelos idosos. Estudos Interdisciplinares sobre 0 Envelhecimento, 2017; 22(3): 153-167.

9. FUNDAÇÃO GETÚLIO VARGAS (FGV). Mapa da inclusão Digital. Rio de Janeiro: Fundação Getúlio Vargas, 2012. Disponível em: https://bibliotecadigital.fgv.br/dspace/bitstream/handle/10438/20738/Sumario-Executivo-Mapa-daInclusao-Digital.pdf. Acesso em: 22/09/2021

10. GARBIN HBR, et al. A internet, o paciente expert e a prática médica: uma análise bibliográfica. Interface Comunicação, Saúde, Educação, 2008; 12(26): 579-588.

11. GUSTAFSON SR DH, et al. A Web-Based eHealth Intervention to Improve the Quality of Life of Older Adults With Multiple Chronic Conditions: Protocol for a Randomized Controlled Trial. JMIR Res Protoc, 2021b; 10(2): e25175.

12. GUSTAFSON SR DH, et al. Effect of an eHealth intervention on older adults' quality of life and health-related outcomes: a randomized clinical trial. J Gen Intern Med, 2021a; 7: 1-10.

13. GUSTAFSON SR DH, et al. The effect of an information and communication technology (ICT) on older adults' quality of life: study protocol for a randomized control trial. Trials, 2015; 16: 191.

14. INSTITUO BRASILEIRO DE GEOGRAFIA E ESTATÍSTICA (IBGE). Projeções da População: Brasil e Unidades da Federação Revisão 2018, 2018a. Disponível em: https://biblioteca.ibge.gov.br/visualizacao/livros/liv101597.pdf. Acesso em: 7 set. 2021.

15. INSTITUTO BRASILEIRO DE GEOGRAFIA E ESTATÍSTICA (IBGE). Pesquisa nacional por amostra de domicílios contínua: acesso à internet e posse de telefone móvel celular para uso pessoal: 2018b. Disponível em: https://www.ibge.gov.br/estatisticas/sociais/populacao/17270-pnad-continua. html?edicao=23205\&t=sobre. Acesso em: 7 set. 2021

16. KNORST GRS, et al. Doctor-patient relationships in the era of the expert patient: An epistemological analysis. Interface: Communication, Health, Education, 2019; 23: e180308.

17. KRUG R, et al. Associação entre o uso de internet e a função cognitiva de idosos, estudo longitudinal populacional Epifloripa Idoso. Revista Brasileira de Epidemiologia, 2019; 22: e190012.

18. LEGER D, et al. Prevalence of insomnia in a survey of 12,778 adults in France. Journal of Sleep Research, 2000; $9(1)$ : 35-42.

19. LIPSCHITZ DA. Screening for nutritional status in the elderly. Primary Care, 1994; 21(1): 55-67.

20. MASNOON N, et al. What is polypharmacy? A systematic review of definitions. BMC Geriatr, 2017; 17(230).

21. MEISCHKE H, et al. Do older adults use the internet for information on heart attacks? Results from a survey of seniors in King County, Washington. Heart Lung, 2005; 34(1): 3-12.

22. MINISTÉRIO DA SAÚDE. Secretaria de Atenção à Saúde. Departamento de Atenção Básica. Guidelines for evaluation of food consumption markers in Primary Health Care. Brasília: Ministério da Saúde, 2016. Disponível em: http://bvsms.saude.gov.br/bvs/publicacoes/orientacoes_avaliacao_marcadores_consumo_alimentar_atencao_basica .pdf. Acesso em: 7 set. 2021.

23. MIRANDA GMD, et al. Population aging in Brazil: current and future social challenges and consequences. Revista Brasileira de Geriatria e Gerontologia, 2016; 19(3): 507-519.

24. MIRANDA LM, FARIAS SF. Contributions from the internet for elderly people: a review of the literature. Interface Comunic., Saúde, Educ., 2009; 13(29): 383-94.

25. MURRAY E, et al. Interactive health communication applications for people with chronic disease. Cochrane Database of Systematic Reviews, 2005; 4: 1-82.

26. NETO AP, FLYNN MR. Internet e Saúde no Brasil: Desafios e Tendências. 1ed. São Paulo: Cultura Acadêmica Editora, 2021, 459p.

27. NEVES BB, et al. Social capital and Internet use in an age-comparative perspective with a focus on later life. PLOS ONE, 2018; 13(2): e0192119.

28. PAIM J, et al. The Brazilian health system: history, advances, and challenges. The Lancet, 2011; 377: $1778-1797$.

29. PANDEY SK, et al. Women's health and the internet: Understanding emerging trends and implications. Social Science and Medicine, 2003; 56(1): 179-191.

30. QUITTSCHALLE J, et al. Internet use in old age: results of a german population representative survey. J Med Internet Res, 2020; 22(11): e15543.

31. SAMKANGE-ZEEB F, et al. Superdiversity, migration and use of internet-based health information - Results of a crosssectional survey conducted in 4 European countries. BMC Public Health, 2020; 20(1): 1263.

32. SANTOS AF, et al. Incorporação de Tecnologias de Informação e Comunicação e qualidade na atenção básica em saúde no Brasil, 2017; 33(5): e00172815.

33. VICTORA CG, et al. The role of conceptual frameworks in epidemiological analysis: A hierarchical approach. International Journal of Epidemiology, 1997; 26(1): 224-227.

34. WANG L, et al. The Health Status Transition and Medical Expenditure Evaluation of Elderly Population in China. Int J Environ Res Public Health, 2021; 18(13): 6907. 